\title{
A Hand-Eye Robotic Model for Total Knee Replacement Surgery
}

\author{
Fanhuai Shi ${ }^{1}$, Jing Zhang ${ }^{1}$, Yuncai Liu ${ }^{1}$, and Zijian Zhao ${ }^{2}$ \\ ${ }^{1}$ Inst. Image Processing \& Pattern Recognition, \\ Shanghai Jiao Tong University, \\ Shanghai 200030, P.R. China \\ \{fhshi, zhjseraph, whomliu\} esjtu.edu.cn \\ ${ }^{2}$ School of Information Science \& Engineering, Shandong University, \\ Jinan 250100, P.R. China \\ zhaozijian@sdu.edu.cn
}

\begin{abstract}
This paper presents a hand-eye robotic model for total knee replacement (TKR) surgery. Unlike existent robot assisted TKR surgery, the proposed model is a surgical robot that combines with a movable hand-eye navigation system, which would use the full potential of both computer-assisted systems. Without using CT images and landmark pins in the patient's bones, it can directly measure the mechanical axis with high precision. This system provides a new approach of the minimally invasive surgery. Experiment results show that the proposed model is promising in the future application.
\end{abstract}

\section{Introduction}

Total knee replacement (TKR) surgery is a common orthopaedic procedure to replace damaged articular surfaces of the knee with prosthetic implants. To fit the prosthesis, each of the knee bones (tibia, femur and patella) should be cut to a specific shape to mate the mounting side of the corresponding prosthesis component. To ensure normal functionality of the knee, all components must be placed onto the bones with high precision. For this aim, we must consider both the bone axes and the mating surfaces between the bone and prosthesis [1].

During the operation, traditionally, a complex jig system composed of cutting blocks, alignment rods, etc., is used to help the surgeon to estimate the geometry of the bones and select the appropriate size and location of the components. The accuracy of this process relies on the surgeon's individual experience. Besides, the traditional jig-based systems also introduce several sources of inaccuracy in alignment of the prosthetic components [2]. The limitations of traditional knee surgery have prompted the research for a more accurate and repeatable system for TKR.

The rapid development of robotics provides a new approach to improve the surgery quality. Due to the rigid nature of bone, it is relatively easy to image in computed X-ray tomography (CT) and X-ray fluoroscopy [3]. So orthopaedics suits for robotic assistance. Moreover, robot assisted TKR can enhance the quality of the bone cuts and require less time for surgery [2]. 


\subsection{Related Work}

There are already commercially available surgical robots for joint replacement surgery, such as ROBODOC [4] and CASPAR [5]. Both systems adapted industrial robots for surgical task and were initially aimed at hip replacement. Unfortunately, they still require additional surgery to preimplant fiducial markers. ROBODOC recently started using an anatomical registration procedure [6]. Researchers in Imperial College, London developed a "hands-on" robot, called Acrobot, for TKR surgery [1]. Intraoperatively, the surgeon guides the special-purpose robot, which is mounted on a gross positioning device. Acrobot uses active constraint control to constrain the motion of the cutter to a predefined region, and thus allows the surgeon to safely cut the knee bones with high precision. Nevertheless, all the above systems need to take plenty of CT images for registration.

The technology of knee replacement is evolving, changing, and improving. Laskin and Richard [7] described some new techniques and concepts in TKR, especially Minimally Invasive Surgery (MIS). A good approach for MIS is the use of a CT-free image-guided system, which usually based on optically tracked surgical tools with a visual display of the bone models to aid in the positioning and alignment of tools. Aesculap Inc. has developed Unveils OrthoPilot, which is the industry's first CT-free navigation system for orthopedic surgery and has been routinely used in orthopaedic surgery [8]. Other commercialized systems include BrainLab's (BrainLab, Germany), Stryker Leibinger's (Leibinger, Germany), and PiGalileo (PLUS Orthopedics USA, Inc.). These navigation systems, however, while helping to improve accuracy, should not be moved during the operation, which greatly constrains the field of view of the optic sensors. In brief, we call such system as static navigation system. More recently, DiGioia et al. provided rich review of these relevant technologies in [9].

\subsection{Contributions of This Paper}

In this paper, we propose a hand-eye robotic model for TKR surgery. In this model, both the optic sensors and the cutting tool are fixed on the end-effecter/gripper of a surgical robot, which can be moved freely to anywhere within the capability of the robot. Thus, we combine the surgical robot and navigation system together, which would use the full potential of both computer-assisted systems. As the navigation system can move while the robot gripper is moving, we call it a movable navigation system. The remainder of this paper decomposes as follows. Section 2 recalls the target problem in TKR surgery. Then the proposed model is presented in detail in section 3 . Section 4 describes how to use the proposed model for TKR surgery. Some experiments on performance test are conducted in section 5 .

\section{Problem Formulation}

Let's revisit the target problem in TKR surgery. Shown as Fig. 1(a), the normal mechanical axis of the leg is formed by a straight line starting from the center of the femoral head $B$, passing through the center of the knee joint $O$ and ending at the center of the ankle $C$. The transverse axis passing through the joint is parallel to the floor when 


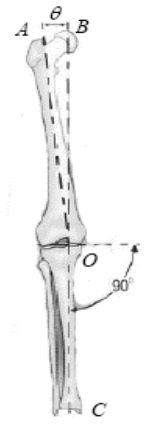

(a)

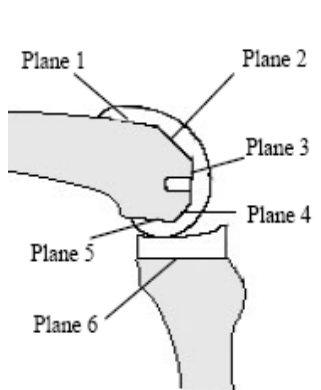

(b)

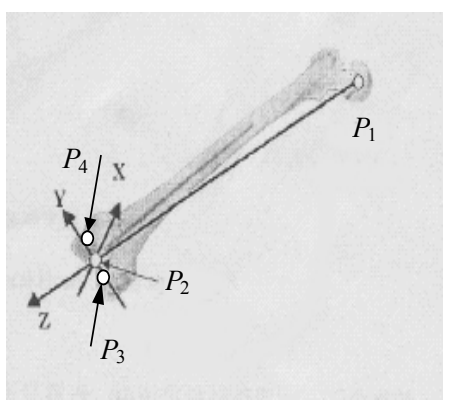

(c)

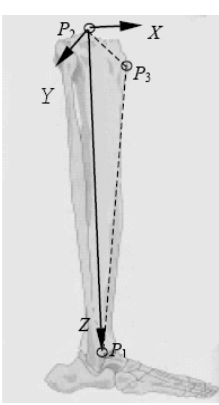

(d)

Fig. 1. Main task of TKR surgery. (a) Restoration of the mechanical axis. (b) Surface of the bone to be cut. (c) Femoral positioning and measure. (d) Tibia positioning and measure.

one stands. The angle $\theta$ between the normal mechanical axis $O B$ and the axis of the femur canal $O A$ is about $5^{\circ} \sim 9^{\circ}$ [10]. The aim of TKR surgery is to restore the normal mechanical axis and create a joint plane, which is vertical to the mechanical axis, while sacrificing minimal bone stock and maximizing collateral ligament. Thus, shown in Fig. 1(b), five planes on the distal femur (plane 1-plane 5) and one plane on tibia (plane 6) must be cut to fit on prosthesis components. Therefore, there are mainly two tasks in the TKR surgery: one is the precise measurement of the mechanical axis and the other is the accurately milling of the fitting plane. The former task is especially dependent on the accuracy of the navigation system.

Unfortunately, the mechanical axis is not directly measurable by the surface of the femoral, tibia, or deforming joint. For example, present methods of measuring $O B$ firstly figure out the axis of the femur canal $O A$, and then make a rotational alignment of $5^{\circ} \sim 9^{\circ}$. This kind of alignment heavily depends on the experience of the surgeons.

\section{Techniques of the New Model}

In this paper, we propose a flexible hand-eye robotic model, in which the navigation system can directly measure the mechanical axis without using CT images or other indirect ways. In contrast to previous navigation system for orthopaedic surgery, which uses static navigation system, a hand-eye, special-purpose robot, called WATO, has been built for a movable navigation and operation in TKR surgery. Besides, the cutting tool is fixed on the end-effecter of the robot, which would use the full potential of both computer-assisted systems.

\subsection{System Configuration}

The WATO is a 6-DOF industrial robot (MOTOMAN) with the cutting tool and a pair of CCD cameras attached on the end-effecter. After the stereo rig is precisely calibrated, we mount an infrared filter on each camera. Thus, we get an infrared stereovision system, see Fig. 2. In order to make precise measuring on the bone, some infrared positioning devices, such as infrared marker and infrared probe, are developed. 


\subsection{Positioning and Measuring in the Surgery}

Positioning and measuring are the most important and difficult technology in TKR surgery. Traditional mechanical guidance relies heavily on an individual surgeon's experience with a given jig system. CT-based image-guidance system has greatly promoted the measure in robot assisted TKR surgery. However, early approach requires an additional surgery to implant several metal pins into the bone and the later developed noninvasive anatomical registration method is time-consuming.

In WATO system, neither CT models nor metal markers on the patient's bone are needed. Instead, an infrared guide system is used which consists of a binocular vision system and its associated infrared marker/probe. Several special infrared emitters are embedded in the infrared probe, whose 3-D coordinates can be detected by the binocular vision system. After the infrared probe calibration, the position of the probe tip relative to those

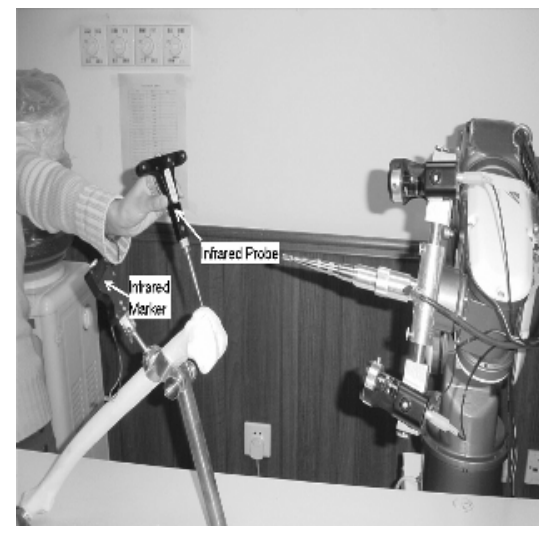

Fig. 2. WATO Experimental System diodes can be obtained. Therefore, when the probe picks up a space point, the position of infrared diodes can be detected and then the position of the probe tip. To build femoral and tibia coordinate system, surgeons are requested to detect some physiological marks on patient's bone by probe. Fig. 2 shows a probe that was used in WATO system. Similar to infrared probe, the infrared marker also consists of some infrared emitters and is clamped on the distal femur or tibia. According to the relationship between the marker and the bone, the motion of the bone can be monitored by the vision system. We will explain it in detail in the following.

Femoral measure. Sufficient surgical exposure is needed to fix the infrared marker, surgeons instead of robot complete this procedure. After surgical exposure, we clamp an infrared marker on the distal femur, see Fig. 2.

In the jig-based surgery, see Fig. 1(a), a baseline axis $A O$ is first measured by inserting a T-rod into the femoral canal. Then they can restore the mechanical axis $B O C$ by adjust $A O 5^{\circ} \sim 9^{\circ}$. In WATO system, however, the axis $B O C$ can be acquired directly. Shown as Fig. 1(c), $P_{1}$ is the center of the femoral head, $P_{2}$ is the center of intercondylar fossa, $P_{3}$ is the epicondylus medialis, and $P_{4}$ is the epicondylus lateralis. With the infrared marker clamped on the femur, the surgeon manually flexes and abducts the entire leg (which is able to rotate only about the femoral head) through substantial arcs, while the hand-eye navigation system observing the position of the infrared marker. As the detected positions of the infrared emitters are on a sphere whose center is also the center of the femoral head, then we can obtain the coordinate of $P_{1}$. It is important that the surgeon must take special care during the pivot motion with the femur and not to move the pelvis, which would invalidate the pure rotation. This 
approach is inspired by the work of Kienzle et al. [2]. Meantime, the coordinates of $P_{2}, P_{3}$ and $P_{4}$ can be obtained by infrared probe. Straight line $P_{1} P_{2}$ is equivalent to the mechanical axis $B O C$ in Fig. 1(a). Take the center of intercondylar fossa $P_{2}$ as the origin, $\overrightarrow{P_{1} P_{2}}$ as the direction of $Z$ axis, the cross product of the vectors $\overrightarrow{P_{3} P_{4}}$ and $\overrightarrow{P_{1} P_{2}}$ as the direction of $X$ axis. Then one can obtain the direction of $Y$ axis by cross product of $Z$ axis and $X$ axis. Thus, the femoral coordinate system $P_{2}-X Y Z$ is constructed. We denote the rigid transformation between the femoral coordinate system and the camera coordinate system with the couple $\left(R_{f c}, t_{f c}\right)$, and then we have

$$
R_{f c}=\left[\overrightarrow{P_{3} P_{4}} \times \overrightarrow{P_{1} P_{2}}, \overrightarrow{P_{1} P_{2}} \times\left(\overrightarrow{P_{3} P_{4}} \times \overrightarrow{P_{1} P_{2}}\right), \overrightarrow{P_{1} P_{2}}\right], t_{f c}=\vec{P}_{2}
$$

The transformation from camera to gripper $\left(R_{c g}, t_{c g}\right)$ can be obtained by hand-eye calibration [11] in advance and the transformation from gripper to robot base $\left(R_{g r}, t_{g r}\right)$ can be computed from the recorded gripper pose. After constructing femoral coordinate system, we can obtain the relationship $\left(R_{f r}, t_{f r}\right)$ between the femur and the robot coordinate system by

$$
\left[\begin{array}{cc}
R_{f r} & t_{f r} \\
0 & 1
\end{array}\right]=\left[\begin{array}{cc}
R_{g r} & t_{g r} \\
0 & 1
\end{array}\right]\left[\begin{array}{cc}
R_{c g} & t_{c g} \\
0 & 1
\end{array}\right]\left[\begin{array}{cc}
R_{f c} & t_{f c} \\
0 & 1
\end{array}\right]
$$

Therefore, any point $\left(x_{f}, y_{f}, z_{f}\right)^{T}$ in the femoral coordinate system can be transformed to the coordinate $\left(x_{r}, y_{r}, z_{r}\right)^{T}$ in robot base as

$$
\left(x_{r}, y_{r}, z_{r}\right)^{T}=R_{f r} \cdot\left(x_{f}, y_{f}, z_{f}\right)^{T}+t_{f r}
$$

In making surgical plans, a planning software is used to interactively decide prosthesis size according to the sampled data of individual patient's femur. Then the placement of the prosthesis and cutting quantity could be estimated automatically. All the data in femoral coordinate system are transformed to the data in robotic coordinate system, then the robot are controlled to perform bone resection with special designed cutting tool.

Tibia measure. Similar to femoral positioning, as shown in Fig. 1(d), surgical exposure of the tibia is made by surgeons in advance and then the infrared marker is clamped onto the distal tibia. Since the mechanical axis passes through the center of the knee joint and ends at the center of the ankle, tibia coordinate system must be constructed in accordance with this axis. Firstly, the positions of the physiology fiducial mark of malleolus medialis and malleolus lateralis are sampled by using infrared probe. We assume that the mid-point of the two points is the center of ankle joint. In the similar way, surgeons can obtain the position of $1 / 3$ part on the tuberculum intercondylare mediale $\left(P_{2}\right)$ and tuberositas tibiae $\left(P_{3}\right)$. Define the plane $P_{1} P_{2} P_{3}$ formed by tuberculum intercondylare mediale, center of ankle and tuberositas tibiae. We define the direction of vector $\vec{P}_{2} P_{1}$ as the direction of $Z$ axis. The line in plane 
$P_{1} P_{2} P_{3}$ which is perpendicular to $Z$ axis is defined as the direction of $X$ axis. Then $Y$ axis is the cross product of $Z$ and $X$ axis. So we have constructed tibia coordinate system. After constructing tibia coordinate system, we can obtain the relationship between the two coordinate systems of tibia and infrared marker. When the infrared marker is combined with the tibia, the pose monitoring and resection of it are similar to the way of femur.

\subsection{Visual Servoing in the Surgery}

In the surgery, the milling track of the cutting tool can be planned in advance. However, in conventional system, patient is not fixed completely during the whole operation. Consequently, when unwanted micro-movements of the leg occurs during robotic surgery, the surgical plan must be re-designed, which is time-consuming. In order to deal with this problem, the traditional method is trying to fix the patient's leg and an alarming device is mounted on the patient's leg. For example, in ROBODOC TKR system [6], bony spiculas are fitted into the femur and tibia to guarantee the patient's leg unmovable. However, in TKR surgery, it often requires a different flexion angle of the knee in order to find an optimal pose for operation. Once the patient's leg is fixed completely, the surgeons will have some trouble in operation especially in an emergency. All the above disadvantages constrain the applications of such systems.

The above problems have been solved in WATO system when we fix the infrared markers onto the bones. During the surgery, the passive markers are constantly monitored by the cameras, so does the associated femur or tibia coordinate system. In this way, a position-based visual servoing system [12] comes into being. In CASPAR system, the movement of the leg is also monitored by an infrared camera system [5], however, it is based on a static navigation system. When the navigation system keeps tracking the position and orientation of the infrared marker (or the bone), the movement of the bone will not affect the bone resection, which guarantee a safe operation. Besides, as the position-based visual servo in the system needs only simple stereo computation, it performs much faster than that of registration between CT model and the patient's bone. The refresh time of the visual servoing system is less than 0.2 second, which can fulfill the surgical requirement.

\section{System Operation}

To apply our model to TKR surgery, the basic steps are as follows.

\section{Preoperative procedures}

1. Mount two cameras and the cutting tool (a milling cutter) on the end-effector/gripper of the robot (see Fig. 2). Calibrate the stereo rig (camera calibration) and the transformation relationship between the robot gripper and the stereo rig (hand-eye calibration).

2. Mount an infrared filter on each camera. Calibrate the infrared probe using the stereo rig and find the 3-D position of the probe tip in the probe coordination system.

3. Carry out the sterilize process. 


\section{Surgical procedures}

1. Immobilize the pelvis using specially designed fixtures and clamped the infrared markers onto the distal femur. Find the center of the femoral head using the method described in section 3.2.

2. Use the infrared probe to determine the coordinate of the physical fiducial marks on the distal femur in order to construct the femur coordinate system.

3. Use planning software to interactively decide the prosthesis size and placement.

4. Use the robot to guide the surgical cuts for placement of the femoral component.

5. Clamp the infrared marker on the tibia and use the similar method as that of femur to construct the tibia coordinate system.

6. Use the robot to guide the surgical cuts for placement of the tibia component.

\section{Performance Test of the Navigation System}

The hand-eye navigation system is the most distinctive part in the proposed model. In this section, we test the performance of the navigation system with two different measurement schemes. First, a fixed infrared marker was used to test the calibration accuracy of the stereo rig and the hand-eye relationship. In the second test, we use a calibrated infrared probe to sample the crossings of a chessboard, which will test the positioning accuracy of the infrared probe, see Fig. 4.

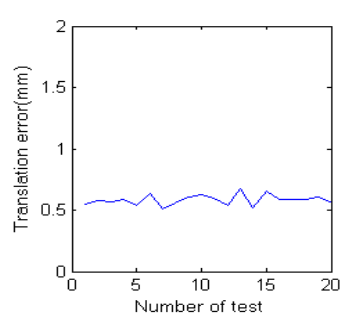

(a)

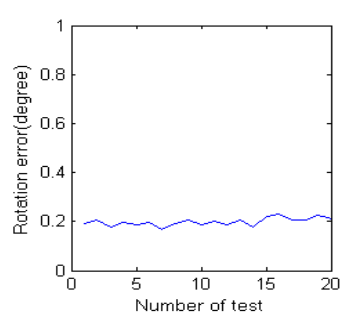

(b)

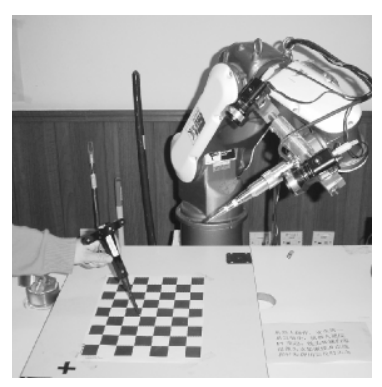

Fig. 4. Positioning test with the infrared probe

Fig. 3. Results of the first test. (a) Mean error of the three translation components; (b) Mean error of the three rotation components.

In the first test, the robot gripper is randomly moved to 30 different positions to observe a static infrared marker and each recorded gripper pose is denote as $P_{i}=\left(X_{i}, Y_{i}, Z_{i}, R X_{i}, R Y_{i}, R Z_{i}\right), i=1,2, \ldots, 30$; repeat the test for 20 times. $X_{i}, Y_{i}, Z_{i}$ are the translation components and $R X_{i}, R Y_{i}, R Z_{i}$ are the three pose angles of robot gripper. Then camera motion $B_{j}(j=1,2, \ldots, 29)$ between frame $i$ and frame $i+1$ can be calculated according to pose of the infrared marker. Then, from the basic equation of robotic hand-eye relationship $A_{j} X_{0}=X_{0} B_{j}[11]$, where $X_{0}$ is the relation between robotic gripper and cameras, the gripper motion $A_{j}$ can be obtained. Notice that $P_{i+1}=P_{i} A_{j}$, we can obtain a sequence of calculated robotic positions $\tilde{P}_{i+1}$. We compare the translation part and rotation angle of $P_{i+1}$ with that of $\tilde{P}_{i+1}$ respectively. Shown as 
Fig. 3, the mean error is illustrated in solid line, in which mean error of the translation part is $0.58 \mathrm{~mm}$, the standard deviation is $0.44 \mathrm{~mm}$, while rotation angle is 0.19 degree and 0.17 degree respectively.

In another test, a calibrated infrared probe is used to sample the crossings of a chessboard, where the real distance $d$ between crossings is known. The distance between crossings that calculated from the 3-D coordinates sampling by the probe is $d$ '. Here, the coordinates sampled by the probe are all in the camera coordinate system. We randomly move the chessboard to 10 locations with different translation and orientation while keeping the cameras or the robot gripper static. In each location, 40 pairs of crossings are sampled. To qualify the results, we take RMS of the error $\| d-d$ 'll. The mean error is $0.40 \mathrm{~mm}$.

From the experimental results, we can see that a goal of less than $0.6 \mathrm{~mm}$ of translational error and less than 0.2 degree of rotational error is achievable, which means that WATO can fulfill the requirement of the TKR surgery.

\section{Summary and Future Work}

A new robot assisted surgical model (WATO) is proposed for TKR in this paper. Unlike existent solutions, WATO is a surgical robot that combines together with a movable, hand-eye navigation system. Without using CT images and landmark pins in the patient's bones, WATO can directly measure the mechanical axis with high precision, which affords a new approach for the development of the minimally invasive surgery. Experimental results show that WATO is promising in the future application.

However, there are still some places to be improved. The present infrared marker needs too much surgical exposure to fix on the femur, which increases the invasiveness of the procedure. So, we need to design more efficient clamps to fix the infrared marker. In addition, as the proximity of the camera to the cutting tool, some splash-guard and vibration control mechanism should be considered in the future.

\section{References}

[1] Jakopec M., Baena F. R. Y., Harris S. J., Gomes P., Cobb J., and Davies B. L.: The Hand-On Orthopaedic Robot 'Acrobot': Early Clinical Trials of Total Knee Replacement Surgery. IEEE Trans. Robot. Automat. 19(5):902-911,2003.

[2] Kienzle, T.C., III; Stulberg, S.D.; Peshkin, M.; Quaid, A.; Lea, J.; Goswani, A.; Wu, C.-h.: Total Knee Replacement. IEEE Engineering in Medicine and Biology Magazine, 14(3): 301-306, 1995.

[3] Taylor R. H. and Stoianovici D.: Medical Robotics in Computer- Integrated Surgery. IEEE Trans. Robot. Automat. 19(5):765-781, 2003.

[4] Taylor R. H., Paul H.A., Kazandzides P., Mittelstadt Hanson B. D.,W., Zuhars J. F., Williamson B., Musits B. L., Glassman E., and Bargar W. L.: An image-directed robotic system for precise orthopaedic surgery. IEEE Trans. Robot. Automat., vol. 10, pp. 261-275, Apr. 1994.

[5] Siebert W., Mai S., Kober R., Heeckt P.F.: Technique and first clinical results of robot-assisted total knee replacement. The Knee. 9(3): 173-180, 2002. 
[6] Wiesel U., Lahmer A., Tenbusch M., and Borner M.: Total knee replacement using the ROBODOC system. in Proc. 1st Annu. Meeting CAOS Int., Davos, Switzerland, 2001, p. 88.

[7] Laskin, Richard S. MD.: Session IV, New Techniques and Concepts in Total Knee Replacement. Clinical Orthopaedics \& Related Research. 1(416): 151-153, November 2003.

[8] Sundaraj, K.; Laugier, C.; Boux de Casson, F.: Intra-operative ct-free examination system for anterior cruciate ligament reconstruction. Proc. IEEE/RSJ International Conference on Intelligent Robots and Systems, Oct. 2003, Vol. 3, pp:2829-2834.

[9] DiGioia A. M., Jaramaz B., Picard F., and Nolte L. P.: Computer and Robotic Assisted Hip and Knee Surgery. Oxford University Press, 2004.

[10] Gray M. Ferguson, M.D., Richard J. Friedman, M.D. and FRCS(C): Foundation Knee System Primary Surgical Technique, Encore Orthopedics, Inc. 1999.

[11] Andreff N., Horaud R., and Espiau B.: Robot hand-eye calibration using structure from motion. Int. J. Robot. Res., 20(3):228-248, 2001.

[12] Hutchinson, S., Hager, G. D., and Corke, P. I.: A tutorial on visual servo control. IEEE Trans. Robot. Automat. 12(5):651-670, 1996. 